\title{
No correlation between pinopode formation and LIF and MMP2 expression in endometrium during implantation window
}

\author{
Mateusz Mikołajczyk, Jana Skrzypczak, Przemysław Wirstlein \\ Division of Reproduction, K. Marcinkowski University of Medical Sciences, Poznan, Poland
}

\begin{abstract}
Implantation depends on two factors - embryo and endometrium. The period of maximal endometrial receptivity is a poorly understood phenomenon. We decided to look at three possible markers of implantation: pinopodes, leukemia inhibitory factor, and matrix metalloproteinase 2 and their correlations. We included in the study 23 idiopathic infertility patients and 21 patients with recurrent spontaneous abortions of unknown etiology. Twenty one fertile patients were also recruited. A biopsy was used for endometrial dating according to the Noyes and Hertig criteria, and assessed for the presence of pinopodes via a scanning electron microscope. Endometria were examined in Real Time-Polymerase Chain Reaction cycles for the mRNA expression of leukemia inhibitory factor (LIF) and matrix metalloproteinase 2 (MMP2). No difference was found in the stage of pinopodes development, nor in the coverage of endometrial surface between the studied groups. The expression level for LIF mRNA was lower in control patients compared to idiopathic infertility and recurrent miscarriage patients. No difference was detected in the expression of MMP2 between all studied groups. No correlation was found between pinopodes development stage and LIF and MMP2 expressions in endometrium. Of the studied factors, LIF and pinopodes show the most promise as potential markers of endometrial receptivity. However, the results achieved suggest that these markers are independent of each other. (Folia Histochemica et Cytobiologica 2011; Vol. 49, No. 4, pp. 615-621)
\end{abstract}

Key words: infertility, recurrent miscarriage, pinopodes, endometrium, LIF, MMP, idiopathic infertility

\section{Introduction}

Successful implantation requires proper development of both the embryo and the endometrium. While we have methods to describe the development of an embryo either by morphologic indices or genetic screening, a proper description of endometrial receptiveness still eludes us $[1,2]$. There are many potential markers of the so-called 'implantation window' but most of them have proven to be redundant in the implantation processes [3]. The search for markers had been chaotic at best, until the introduction of gene

Correspondence address: K. Wirstlein,

Division of Reproduction, K. Marcinkowski

University of Medical Sciences,

Polna Str. 33, 60-535 Poznan, Poland;

tel.: 618419 302, fax: 618419625 ;

e-mail: abys@wp.pl chip matrices that allow simultaneous analysis of many thousands of genes. This in turn enabled Kao et al. to build a road map of genes that are up- and down regulated during the implantation window [4]. However, some of these genes could be redundant, or replaced by other genes, while others might be indispensable in the reproductive process of humans. Therefore it is up to scientists to study single genes and their expression in the period of maximal receptivity in patients to find out which are true markers of uterine receptivity.

We decided to look at three potential markers of the receptive phase in patients with infertility, recurrent spontaneous abortions and fertile controls.

The first of these markers are pinopodes. These are apical, bulbous projections of the endometrium that are said to have some pinocytic function in other species, hence the name [5]. However, their true role in the implantation process in humans remains un- 
known, as are the mechanisms behind their appearance $[6,7]$. Some researchers say that pinopodes are present on the surface of the endometrium for no more than 48 hours during the implantation window, sharply delineating the period of maximal receptivity. Yet others claim that pinopodes can be found for up to seven days in the second phase of the menstrual cycle $[8,9]$. Also, research has pointed to a possible relationship between impaired fertility and decreased or dissynchronous appearance of pinopodes, although this view is not shared by all scientists $[10,11]$.

The second marker of uterine receptivity appears to be Leukemia Inhibitory Factor (LIF). This was first discovered in 1987, and in 1992 its crucial role in implantation in mice became evident [12]. There have since been numerous attempts to correlate the decreased expression of this protein with states of impaired fertility in humans [13]. Some researchers have found decreased expression of this protein in patients with recurrent spontaneous abortions and patients with infertility, while others have failed to detect such a difference $[14,15]$. Aghajanova et al. correlated the spatial and temporal expression of pinopodes and LIF in human endometrium during the implantation window [16]. It would be interesting to find out whether disturbances in this correlation might be evident in patients with infertility and RSA as opposed to fertile controls.

Another factor important in tissue remodeling during implantation, and possibly responsible for the appearance of pinopodes, is matrix metalloproteinase 2 (MMP2). MMP2, with the TGF superfamily, MMP9 and tissue inhibitors of metalloproteinases (TIMP), regulates together with the embryo the depth of invasion into the endometrial tissue $[17,18]$.

We decided to look at the abovementioned markers in patients with infertility, recurrent spontaneous abortions and fertile controls, as they seem to be interconnected and their true function in implantation is not certain.

\section{Material and methods}

Patients. The study was conducted in the Division of Reproduction, Department of Obstetrics and Gynecology of K. Marcinkowski Medical University in Poznan, Poland between January 2008 and February 2009. We included in the study only those infertile patients in whom either all diagnostic tests were negative, or who presented only with minimal endometriosis. The following tests were performed on each couple: semen analysis, ovulation tracking, hysterosalpingography, hormone studies (including FSH, prolactin and progesterone) and laparoscopy with hysteroscopy. We gathered 23 idiopathic infertility patients and 21 patients with recurrent spontaneous abortions. The mean duration of infertility in each group was 3.4 years (1-5 years) and 3.2 years (1.2-6 years), respectively. Patients with recurrent spontaneous abortion (defined as three consecutive miscarriages) after negative HSG, trombophilia panel (protein C, $\mathrm{S}$, hyperhomocyteinemia, V Leiden mutation, prothrombin mutation and antiphospholipid antibodies), normal ultrasound and no disturbances in $\mathrm{LH}$, prolactin, progesterone and estradiol levels, were also included in the study.

Also 21 healthy patients, matched for age, with at least one child, and who had experienced no miscarriages, and had a negative history of infertility and endometriosis, were enrolled in the study. Those patients were admitted to the hospital for non-endometrial-linked diseases, and were approached to donate the endometrium. The study protocol was approved by the local ethical committee, and the patients signed an informed consent form. No patients in the study or control group had taken any hormonal preparations for at least three months prior to the study.

All patients from the current study (both studied and control groups) had a biopsy sample taken 7-9 days after ovulation, confirmed by ultrasound follicular tracking.

Reverse transcription and real-time PCR. The endometrial sample for qPCR was placed in RNAlater solution from Qiagen GmbH (Hilden, Germany) and frozen until extraction. RNA specific primers for real-time PCR were created with Primer3 software [19] based on mRNA sequence from the NCBI Gene database [20]. The specificity of constructed primer was checked against the BLAST database [21]. Specificity and length of these products was also confirmed on agarose gel.

The following primers were used: for GAPDH - forward: 5'-ACAGTCAGCCGCATCTTCTT-3' and reverse: 5'-ACGACCAAATCCGTTGACTC-3', for LIF - forward: CAGTGGCCAAGTTAATTCC-3' and reverse: 5'-CATTGTCGACTTCCAGAC-3', and for MMP2 - forward: 5'-AAGTATGGCTTCTGCCCTGA-3' and reverse 5'ATTTGTTGCCCAGGAAAGTG-3'.

Total RNA was isolated using a RNAeasy Mini Kit (Qiagen GmbH, Hilden, Germany). For homogenization, QiaShredder columns were used (Qiagen, Germany) according to the manufacturer's instructions. To acquire cDNA, the $1 \mu \mathrm{g}$ RNA was treated with QuantiTect Reverse Transcription (Qiagen, Germany). Quantity at OD $260 \mathrm{~nm}$ and purity at OD $260 / 280 \mathrm{~nm}$ samples was checked spectrophotometrically with NanoDrop ND1000 (ThermoScientific, USA). The reverse transcription reaction was antedated by additional elimination of DNA with the use of the QuantiTect Wipeout buffer, one of the components of QuantiTect Reverse Transcription Kit. The matrix that was achieved was free of any contamination from genomic DNA, amplification of which could falsify the real time PCR reaction.

The resulting $30 \mathrm{ng}$ cDNA was used as matrix for real-time PCR which was conducted on a RotorGene 3000 real- 
-time thermocycler (Corbett Research). The optimized protocol was used with the mastermix including HotStart polymerase (DyNAmo HS SYBRGreen qPCR Kit from Finnzymes (Espoo, Finland).

The thermocycler was set to 40 runs. The qPCR reactions were run in duplicate for each sample. To assess the efficacy of the qPCR reaction, six subsequent 10-times dilutions of DNA was used, that was a specific reaction from the PCR of each studied transcript. The expression of studied LIF and MMP2 was established according to a reference gene, namely glyceraldehyde-3-phosphate dehydrogenase (GAPDH), whose expression in cells is constant across the menstrual cycle.

Scanning microscopy assessment. The second part of the endometrial sample was fixed in $2 \%$ glutaraldehyde in 0.1 M PBS buffer ( $\mathrm{pH} 7.2$ ), dehydrated in a graded ethanol series $(50 \%, 70 \%, 96 \%$, absolute ethanol), critical point dried, and sputter coated with gold. The samples were then examined using a Zeiss Evo 40 scanning electron microscope at $3,500 \times$ magnification. Samples preparation and scanning microscopy assessment were made in the Laboratory of Electron and Confocal Microscopy in the Department of Biology, Adam Mickiewicz University in Poznan. Depending on size and epithelium presentation, probes 5 to 15 scans were done for each sample.

Pinopode scoring and evaluation of developmental stage of endometrial biopsy samples was based on SEM images.
Endometrial tissue was examined under the SEM and assigned a score as previously described [22]. Briefly, scores ranged from 0 to 4 depending on the percentage of the endometrial surface that was covered in pinopodes: score $0(0 \%$ of the apical surface covered in pinopodes), score $1(1 \%$ to $<5 \%)$, score 2 $(5 \%$ to $<10 \%)$, score $3(10 \%$ to $20 \%)$, and score $4(>20 \%)$. Evaluation of developmental stage of endometrial biopsy ranged from 1 to 6 as shown in Figure 1.

The average score from all 100 fields was rounded to the nearest whole number to assign a final score to each biopsy. The same observer scored all biopsies and was blinded to the day of the cycle.

Statistical analysis. For statistical analysis, Kruskal-Wallis One Way ANOVA test was used, and Spearman correlation test where appropriate (Sigma Stat 3.5); $\mathrm{p}<0.05$ was considered statistically significant.

REST2005 software was used to measure the transcription level differences.

\section{Results}

\section{LIF and MMP2 transcript levels}

LIF expression was lower in patients from the control group, while there was no difference in MMP2 transcript level compared to idiopathic infertility patients (Table1).
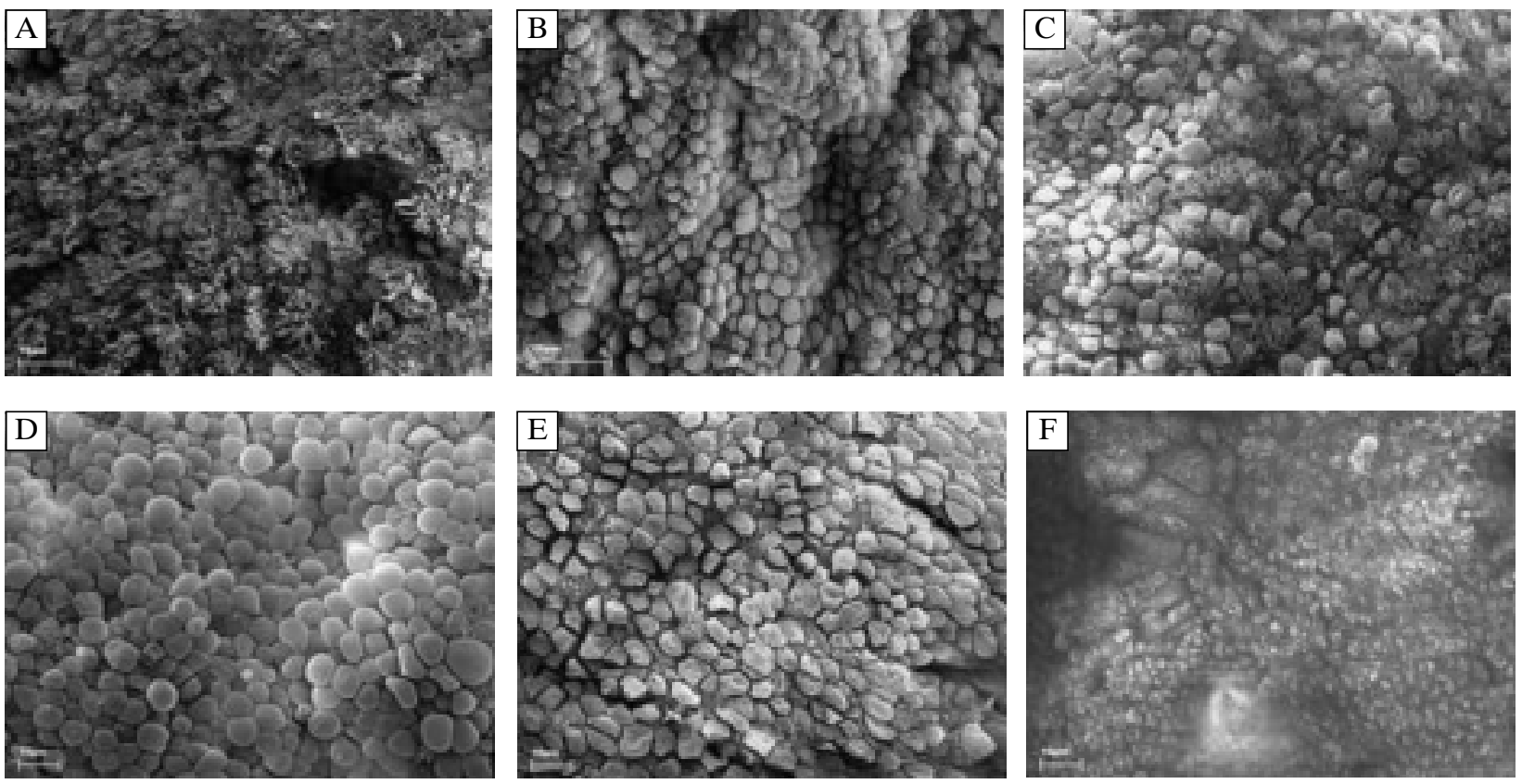

Figure 1. Evaluation of developmental stage of endometrial biopsy samples. Description of phases: A. Before pinopodes develop, the surface starts to bulge and the microvilli become long, thick, and upright. B. Developing pinopodes have protruding smooth surface with or without short microvilli. C. Developing and fully developed pinopodes. D. In fully developed pinopodes, microvilli are absent and the membranes protrude and fold maximally. The shape resembles mushrooms or flowers. E. Regressing pinopodes have wrinkled surfaces, and microvilli reappear on the cell membranes. The cell size starts to increase. F. After pinopodes, the pinopodes and cell bulging regress, and the cell size increases 
Table 1. Relative expressions of LIF and MMP2 in patients with idiopathic infertility vs. controls

\begin{tabular}{|l|c|c|c|c|c|c|}
\hline Gene & Type & Reaction efficiency & Expression & Standard error & 95\% CI & $\mathbf{p}^{*}$ \\
\hline GAPDH & REF & 1.00 & 1.00 & $0.11-15.03$ & $0.0-20147.47$ & 1.000 \\
\hline MMP2 & TRG & 0.89 & 1.87 & $0.26-13.37$ & $0.002-2465.03$ & 0.636 \\
\hline LIF & TRG & 0.89 & 0.06 & $0.01-0.32$ & $0.002-3.91$ & 0.005 \\
\hline
\end{tabular}

CI — confidence interval; *REST analysis

Table 2. Relative expressions of LIF and MMP2 in patients with recurrent spontaneous abortions vs. controls

\begin{tabular}{|l|c|c|c|c|c|c|}
\hline Gene & Type & Reaction efficiency & Expression & Standard error & $\mathbf{9 5 \%}$ CI & $\mathbf{p}^{*}$ \\
\hline GAPDH & REF & 1.00 & 1.00 & $0.07-20.61$ & $0.0-52558.78$ & 1.000 \\
\hline MMP2 & TRG & 0.89 & 2.32 & $0.26-25.1$ & $0.002-4452.26$ & 0.476 \\
\hline LIF & TRG & 0.89 & 0.06 & $0.01-0.26$ & $0.002-20.53$ & 0.004 \\
\hline
\end{tabular}

CI - confidence interval; * REST analysis

Table 3. Relative expressions of LIF and MMP2 in patients with idiopathic infertility and recurrent spontaneous abortions vs. controls

\begin{tabular}{|l|c|c|c|c|c|c|}
\hline Gene & Type & Reaction efficiency & Expression & Standard error & 95\% CI & $\mathbf{p}^{*}$ \\
\hline GAPDH & REF & 1.00 & 1.00 & $0.07-18.36$ & $0.0-33690.17$ & 1.000 \\
\hline MMP2 & TRG & 0.89 & 2.08 & $0.29-18.49$ & $0.002-3868.62$ & 0.453 \\
\hline LIF & TRG & 0.89 & 0.06 & $0.01-0.29$ & $0.002-14.59$ & $<0.001$ \\
\hline
\end{tabular}

CI — confidence interval; *REST analysis

Table 4. Percentage of endometrial surface pinopodes covered

\begin{tabular}{|l|c|c|c|c|c|c|}
\hline Group & $\mathbf{n}$ & Mean & SD & Median & $\mathbf{9 5 \%}$ CI & $\mathbf{p}^{*}$ \\
\hline $\begin{array}{l}\text { Idiopathic } \\
\text { infertility }\end{array}$ & 22 & 2.3864 & 1.6398 & 2.7500 & $1.1072-4.0000$ & 0.175 \\
\hline RSA & 21 & 2.0000 & 1.6733 & 1.0000 & $0.8285-4.0000$ \\
\hline Control & 21 & 3.0000 & 1.2367 & 3.5000 & $1.6912-4.0000$ & \\
\hline
\end{tabular}

SD — standard deviation; CI — confidence interval; *Kruskal-Wallis One Way Analysis of Variance on Ranks

LIF transcript level was lower in patients from the control group, while there was no difference in MMP2 transcript level compared to recurrent spontaneous abortions patients (Table 2).

No difference in transcript levels of LIF and MMP2 was noticed in patients with recurrent spontaneous abortions and infertile patients (Table 3).

\section{Developmental stage of pinopodes and percentage of endometrial surface covered}

In our biopsies, we identified all six stages of pinopode development (Figure 1). No difference in stage of pinopode development (Table 4) nor endometrial surface covered (Table 5) was noticed between the studied groups and the control group.
No correlation between pinopodes development and endometrial covering was noticed in infertile patients (correlation coefficient $-0.31 ; p<0.29$ ), while such a correlation was observed in the fertile control group and the recurrent miscarriage group (correlation coefficient 0.77 ; $\mathrm{p}<0.01$ and $0.59 ; \mathrm{p}<0.05$, respectively).

There was no correlation observed between pinopodes development stage and LIF or MMP2 expression in any of the groups examined.

\section{Discussion}

The implantation period spanning 7-9 days after ovulation is a critical moment, not only the beginning of the pregnancy, but also for its uninterrupted progres- 
Table 5. Developmental stage of pinopodes

\begin{tabular}{|l|c|c|c|c|c|c|}
\hline Group & $\mathbf{n}$ & Mean & SD & Median & $\mathbf{9 5 \%}$ CI & p* \\
\hline $\begin{array}{l}\text { Idiopathic } \\
\text { infertility }\end{array}$ & 22 & 3.6591 & 1.2188 & 4.0000 & $2.6072-4.3928$ & 0.116 \\
\hline RSA & 21 & 3.3095 & 1.1009 & 3.5000 & $2.0000-4.0000$ \\
\hline Control & 21 & 3.9722 & 0.6295 & 4.0000 & $3.6912-4.5000$ & \\
\hline
\end{tabular}

SD — standard deviation; CI — confidence interval; *Kruskal-Wallis One Way Analysis of Variance on Ranks

sion. The mechanisms that govern maximal receptivity are poorly understood in humans. The IVF registers clearly show that implantation is the rate limiting step [23]. While some of the implantation failures might be attributable to a faulty embryo, even with the use of Preimplantation Genetic Diagnostic techniques, the implantation rate remains quite poor [24]. Numerous factors have been hailed as markers of the implantation window, with most of them turning out to be redundant for the receptivity period in humans [3]. However, by careful elimination of such factors, we are getting closer to pinpointing the true determinants of receptivity with possible future treatments aimed at improving this stage in human reproduction.

That is why we chose to study three potential markers of the implantation window, namely: pinopodes, Leukaemia Inhibitory Factor and Matrix Metalloproteinase 2.

Given recent disappointments regarding the role of routine histology of the endometrium in infertile and recurrent miscarriage patients, the attention of scientists has turned to more subtle markers of endometrial dysfunction, like pinopodes $[25,26]$. The exact role of these apical projections is not known, nor their lifespan $[6,7]$. We decided to observe the development of pinopodes in precisely timed biopsies in order to establish whether there were any differences between their stage of development and the percentage of covered luminal surface of the endometrium in infertile patients and women with recurrent miscarriages compared to fertile controls.

There was a correlation between the developmental stage of pinopodes development and the percentage of covered endometrial surface in fertile controls and women with recurrent miscarriages. No such correlation was observed in women with idiopathic infertility. All patients in our study had proper, in-phase endometrial development according to the Noyes and Hertig criteria. Since both the fertile patients and patients with RSA present with normal implantation, this might suggest that proper development of pinopodes, abundant expression of pinopodes on luminal endometrial surface, or both, are required for successful implantation to occur. To find that out, we compared the pinopodes developmental stages in all three studied groups and found no differences. Also there was no difference in the covering of luminal endometrial surface between the groups, which suggests that the effect of pinopodes depends on synchronous development of pinopodes and their coverage of endometrial surface. In such a setting, some patients with infertility despite normal histologic development of endometrium can still suffer from some sort of endometrial dysfunction that renders them infertile. The exact role and function of pinopodes in human endometrium is not known. There are conflicting reports regarding their usefulness as predictors of successful implantation.

The second potential marker of implantation is the Leukemia Inhibitory Factor. It has been stated that LIF deficient mice are totally infertile [12]. Human studies have mostly pointed to a lower LIF expression where infertility and RSA [13, 14] patients are concerned. In our study however, the mRNA expression was lower in the control group compared to the idiopathic infertility and RSA patients. Also no difference was observed in LIF expression between women with infertility and miscarriages. These LIF results are in agreement with a paper published by Lédée-Bataille et al. They described lower LIF concentrations in patients who became pregnant after IVF cycles [27]. These results are contrary to those achieved by other authors and experimental studies where lower LIF concentrations were associated with pregnancy loss or infertility. However, our recently published prospective analysis of pregnancy outcomes in patients with low LIF levels has led to the conclusion that only very low LIF values are needed for pregnancy to occur and to continue to term [28]. It might be argued that higher LIF levels in fact represent a defect in the receptiveness of endometrium, leading to 'overcompensation' in the secretion of certain cytokines by the endometrium. Another possible explanation was offered by Aghajanova et al. who suggested an inverse relationship between LIF and LIF receptor levels [16]. Thus, low LIF expression might correspond to a higher level of LIF receptor, leading to a successful implantation and other changes exerted by LIF glycoprotein on the endometrial cells. 
The third and final marker of the implantation window in humans studied in our current paper was MMP2. MMP2 together with the TGF superfamily are responsible for endometrial tissue remodeling at the time of implantation and menstruation $[17,18]$. Some studies have linked increased expression and activity of MMP2 in endometrium to impairments of implantation resulting in either infertility or recurrent pregnancy loss [17]. As in our previous paper, we found no difference in the expression of MMP2 between any of the groups. We believe that the changes exerted by MMPs and TGF are far more complicated and the possible role of this system deserves further investigation.

Another important aspect of our current study was assessing the possible correlation between stage of formation of pinopodes and LIF mRNA expression. We found no such correlation. Aghajanova et al. found that the expression of fully developed pinopodes was closely correlated with the LIF expression on the endometrial surface [16]. The lack of correlation between the expression of specific stages of pinopode formations and LIF in our paper might suggest that these two markers are independent of each other. On the other hand, similarly to Aghajanova, we also found no correlation between the number of pinopodes and LIF expression. Taken together, we believe that the appearance of pinopodes and LIF are independent of each other and that there is no causative relationship between them. These two markers of implantation simply coexist in the implantation window. Support for our hypothesis came in a recent paper by Quinn et al., who found that the formation of pinopodes was unobstructed by the complete lack of LIF in LIF-deficient mice [22].

A similar situation was encountered as far as MMP2 is concerned. Again, no correlation was observed between MMP2 and the pinopode stage of development, nor the pinopode number. Thus the exact reasons and mechanism behind pinopode development are still undiscovered.

Further research into endometrial receptivity is warranted. Greater knowledge of the events occurring at the blastocyst-endometrial interface could bring us closer to improving implantation rates in IVF cycles. Since many factors might be implicated in the creation of an ideal environment for the implanting embryo, possible correlations between those factors should be examined. Some of the factors, despite their appearance in the implantation window, might turn out to be redundant.

\section{Acknowledgements}

This study was supported by UE grant 162/E-392/CD/ /DFS-4/2004.

\section{References}

1. Pagidas K, Ying Y, Keefe D. Predictive value of preimplantation genetic diagnosis for aneuploidy screening in repeated IVF-ET cycles among women with recurrent implantation failure. J Assist Reprod Genet. 2008;25:103-106.

2. Hesters L, Prisant N, Fanchin R et al. Impact of early cleaved zygote morphology on embryo development and in vitro fertilization-embryo transfer outcome: a prospective study. Fertil Steril. 2008;89:1677-1684.

3. Guzeloglu-Kayisli O, Basar M, Arici A. Basic aspects of implantation. Reprod Biomed Online. 2007;15:728-739.

4. Kao LC, Tulac S, Lobo S et al. Global gene profiling in human endometrium during the window of implantation. Endocrinology. 2002;143:2119-2138.

5. Enders AC, Nelson DM. Pinocytotic activity of the uterus of the rat. Am J Anat. 1973;138:277-299.

6. Martel D, Malet C, Gautray JP, Psychoyos A. Surface changes of the luminal uterine epithelium during the human menstrual cycle: a scanning electron microscopy study. In: de Brux J, Mortel R, Gautray JP (eds). The endometrium: hormonal impacts. NY USA: Plenum Press. 1981:15-22

7. Develioglu OH, Hsiu JG, Nikas G, Toner JP, Oehringer S, Jones HW Jr. Endometrial estrogen and progesterone receptor and pinopode expression in stimulated cycles of oocyte donors. Fertil Steril. 1999;71:1040-1047.

8. Anneli SE, Nikas G, Sahlin L, Eriksson H, Landgren BM. Formation of pinopodes in human endometrium is associated with the concentrations of progesterone and progesterone receptors. Fertil Steril. 2001;76:782-791.

9. Nikas G, Develioglu OH, Toner JP, Jones Jr HW. Endometrial pinopodes indicate a shift in the window of receptivity in IVF cycles. Hum Reprod. 1999;14:787-792.

10. Pantos K, Nikas G, Makrakis E, Stavrou D, Karantzis P, Grammatis M. Clinical value of endometrial pinopodes detection in artificial donation cycles. Reprod Biomed Online. 2004;9:86-90.

11. Bentin-Ley U. Relevance of endometrial pinopodes for human blastocyst implantation. Hum Reprod. 2000;15(Suppl 6): 67-73.

12. Stewart CL, Kaspar P, Brunet LJ et al. Blastocyst implantation depends on maternal expression of leukaemia inhibitory factor. Nature. 1992;359:76-79.

13. Mikolajczyk M, Wirstlein P, Skrzypczak J. The impact of leukemia inhibitory factor in uterine flushing on the reproductive potential of infertile women - a prospective study. Am J Reprod Immunol. 2007;58:65-74.

14. Laird SM, Tuckerman EM, Li TC. Cytokine expression in the endometrium of women with implantation failure and recurrent miscarriage. Reprod Biomed Online. 2006;13:13-23.

15. Steck T, Giess R, Suetterlin MW et al. Leukaemia inhibitory factor (LIF) gene mutations in women with unexplained infertility and recurrent failure of implantation after IVF and embryo transfer. Eur J Obstet Gynecol Reprod Biol. 2004; 112:69-73.

16. Aghajanova L, Stavreus-Evers A, Nikas Y, Hovatta O, Landgren BM. Coexpression of pinopodes and leukemia inhibitory factor, as well as its receptor, in human endometrium. Fertil Steril. 2003;79(Suppl 1):808-814.

17. Skrzypczak J, Wirstlein P, Mikołajczyk M, Ludwikowski G, Zak T. TGF superfamily and MMP2, MMP9, TIMP1 genes expression in the endometrium of women with impaired reproduction. Folia Histochem Cytobiol. 2007;45(Suppl 1):S143-148.

18. Goldman S, Shalev E. Progesterone receptor isoforms profile, modulate matrix metalloproteinase 2 expression in the decidua. Am J Obstet Gynecol. 2007;197:e1-8 [Epub 2007;Aug 6]. 
19. http://frodo.wi.mit.edu/primer3 [Last access 2010;Jul 15].

20. http://www.ncbi.nlm.nih.gov/sites/entrez [Last access 2010; Jul 15].

21. http://www.ncbi.nlm.nih.gov/blast/Blast.cgi [Last access 2010; Jul 15].

22. Quinn C, Ryan E, Claessens EA et al. The presence of pinopodes in the human endometrium does not delineate the implantation window. Fertil Steril. 2007;87:1015-1021 [Epub 2007;Jan 16].

23. Andersen AN, Goossens V, Ferraretti AP et al. European IVF-monitoring (EIM) Consortium; European Society of Human Reproduction and Embryology (ESHRE). Assisted reproductive technology in Europe, 2004: results generated from European registers by ESHRE. Hum Reprod. 2008; 23:756-771 [Epub 2008;Feb 14].

24. Baruch S, Adamson GD, Cohen J et al. Genetic testing of embryos: a critical need for data. Reprod Biomed Online. 2005;11:667-670.
25. McDonough PG. Grading a developmental continuum - elegy on the rise and fall of the endometrial biopsy. Fertil Steril. 2004;82:1286-1292.

26. Pantos K, Nikas G, Makrakis E, Stavrou D, Karantzis P, Grammatis M. Clinical value of endometrial pinopodes detection in artificial donation cycles. Reprod Biomed Online. 2004;9:86-90.

27. Lédée-Bataille N, Laprée-Delage G, Taupin JL, Dubanchet S, Frydman R, Chaouat G. Concentration of leukaemia inhibitory factor (LIF) in uterine flushing fluid is highly predictive of embryo implantation. Hum Reprod. 2002;17: 213-218.

28. Mikolajczyk M, Wirstlein P, Skrzypczak J. The impact of leukemia inhibitory factor in uterine flushing on the reproductive potential of infertile women - a prospective study. $\mathrm{Am}$ J Reprod Immunol. 2007;58:65-74.

Submitted: 25 August, 2010 Accepted after reviews: 1 May, 2011 